\title{
Anterior cruciate ligament recostruction with bone-patellar tendon-bone autograft in Tanner 3 stage patients with open physes
}

\author{
Antonio Memeo ${ }^{a}$, Leopoldo Pedretti ${ }^{b}$, Francesca Miola ${ }^{b}$ and Walter Albisetti ${ }^{b}$
}

\begin{abstract}
Ten skeletally immature patients were treated with an arthroscopic-assisted anterior cruciate ligament reconstruction with bone-patellar tendon bone autograft (compass, 50-55'; holes, 7-9 mm). Radiological assessments (standard radiograph), Orthopädische Arbeitsgruppe Knie (OAK) score and KT 1000, were conducted on all patients, 1 year after surgery. Skeletal maturity had been reached by all patients and no complications were observed. All patients returned to their preinjury sport level. Drilling more vertical tunnels when bone-tendon-bone autograft was chosen to avoid partial epiphysiodesis and offers good functional and isometric
\end{abstract}

results. J Pediatr Orthop B 21:415-420 (c) 2012 Wolters Kluwer Health | Lippincott Williams \& Wilkins.

Journal of Pediatric Orthopaedics B 2012, 21:415-420

Keywords: anterior cruciate ligament, cruciate, injury, ligament, physes, Tanner

astituto Ortopedico G. Pini and ${ }^{\mathrm{b}}$ Department of Orthopaedics and Rehabilitation, Università degli Studi of Milan, Milan, Italy

Correspondence to Leopoldo Pedretti, Department of Orthopaedics and Rehabilitation, Università degli Studi of Milan, viale Gorizia 6, Milano 20100, Italy Tel: + 393395914333; fax: + 3928360546;

e-mail: Leopoldo.pedretti@hotmail.it

MRI of the involved knee. Comparison views of the uninvolved knee and stress view might be required. Hypoplasia of the tibial spine and femoral notch suggests congenital absence of ACL [3,4].

The treatment of ACL injuries in young patients is controversial [4-9]. A skeletally immature patient with open physes requires special attention, as there is a high risk of iatrogenic lesions in the growth plate. Crossing the physes might result in a discrepancy of the leg length or in an angular deformity of the leg [7-9]. Conservative treatment of ACL tears in children has not provided any good outcome $[10,11]$. Although the natural history of ACL tears in skeletally immature patients is characterized by progressive instability, development of meniscal tears, and cartilage damage [12], nonoperative treatment should not be condemned. Conservative treatment might be indicated in children with isolated acute ACL lesion, who will have significant skeletal growth remaining, such as premenarchal girls, and in those individuals who have contraindications or refuse surgery. Conservative treatment includes bracing, quadriceps and hamstring rehabilitation, counseling, and activity modification.

Primary repair of midsubstance ACL tears has shown persistent instability and decreased activity level [13]. Extra-articular procedures might have a role in the treatment of young patients, but the results are not good and not isometric [14]. All epiphyseal reconstructions can be a good option in Tanner 1 patients with important instability, but, to our knowledge, only three cases have been presented [15].

Intra-articular, however, extraphyseal techniques might be used in the treatment of ACL lesion in children and 
adolescents with wide-open physes (Tanner stages 1 and 2). This seems to be a good option [16-20], but some investigators report that the implant is not isometric [21] and anatomic [22]. Although these physeal-sparing techniques have provided relatively good short-term results, the lack of long-term outcomes casts some doubt on the durability and nature of these procedures.

Standard bone tunnels and fixation methods for ACL reconstruction, although common in adults, carry the potential risk for physeal damage in adolescents with open growth plates $[7,8]$. Therefore, the use of transphyseal techniques with fixation of the implant away from the growth plates is well assessed in the literature $[23,24]$. These techniques can be used in pubescent adolescents (Tanner stage 3) with minimal risk of growth disturbance and a low revision rate. However, recent data suggest that drilling across the open proximal tibial or distal femoral physes, or both, can be a safe and effective technique for ACL reconstruction in patients approaching skeletal maturity. Simonian et al. [18] published a review article recommending transphyseal tibial and femoral drill holes. They believe that the use of small, centrally placed tunnels and soft tissue grafts minimizes the risk of physeal closure.

Important radiological studies about the anatomy of the tibial growth plate using three-dimensional MRI and computed tomography scans have been performed during earlier years showing the importance of the diameter of the tunnels and their angle to avoid physeal damage $[25,26]$. The less the diameter and more vertical the tunnel, the lower is the risk.

\section{Materials and methods}

Between 2005 and 2008, 10 skeletally immature patients (eight boys and two girls) were treated for symptomatic instability that had occurred after midsubstance ACL tears. In the majority of the cases, injury was a consequence of sport activity. Mean age at surgery was 14.4 years (range of $13-16$ years). Mean follow-up was 24.9 months (range of 15-44 months).

The evaluation of maturity was performed in all of them before surgical treatment. All the patients presented Tanner stage 3. According to the literature, they could be considered adolescents with growth remaining, presenting an intermediate risk of physeal damage $[16,19]$.

Six injuries involved the left (60\%) and four involved the right knee $(40 \%)$.

Preoperative examination, radiographs, and MRI (Fig. 1) of the knee were obtained in all patients. All patients had radiological evidence of open tibial and femoral physes.

All patients had a positive Lachman and Pivot shift test. All patients underwent intra-articular ACL reconstruction with the same surgical technique and by the same surgeon. The operative procedure comprised an arthroscopic-assisted ACL reconstruction with bone-patellar tendon bone autograft. Surgical technique involved routine superior and lateral notchplasty and removal of residual ACL tissue. Femoral and tibial bone tunnels were drilled to allow anatomic graft position.

The tunnels were drilled slightly more vertically and medially to the anterior tibial apophysis with the compass settled between $50^{\circ}$ and $55^{\circ}$. Holes of $7-9 \mathrm{~mm}$ in diameter were drilled across the proximal tibial and distal femoral physes $(30 \mathrm{~mm}$ of length in the femur) to avoid damaging the open physes $[25,26]$. The grafts were fixed to the distal femur with two pins (rigid-fix system) and to the proximal tibia with an interference reabsorbable screw.

Fig. 1

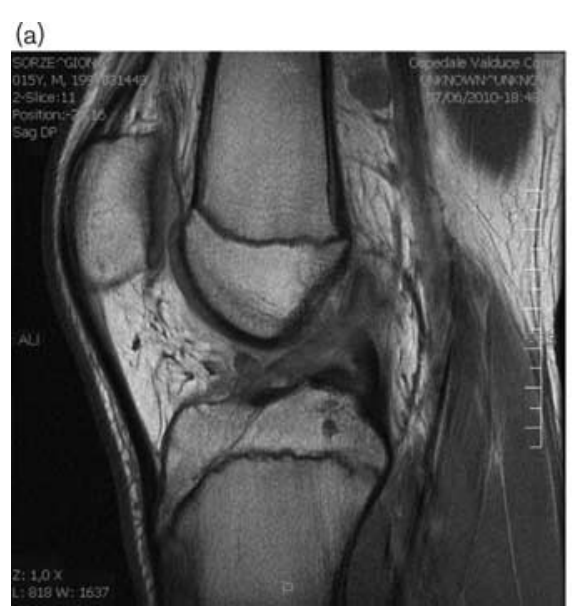

(b)

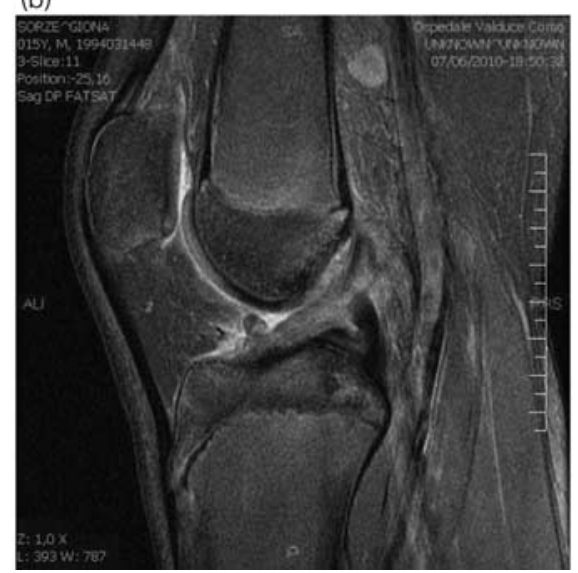

(c)

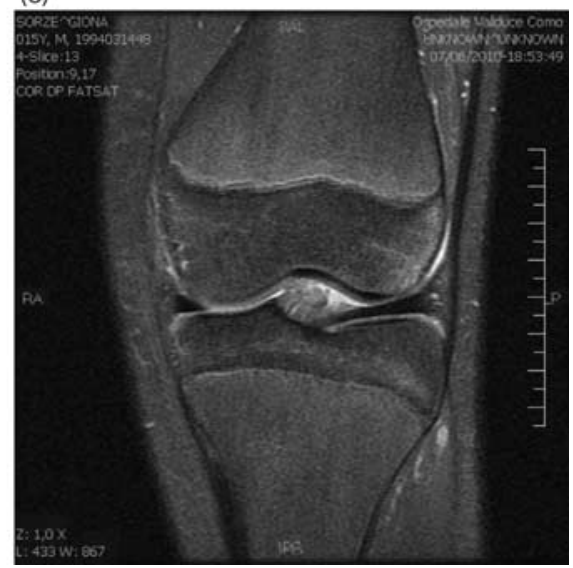

$(a-c)$ Preoperative MRI control showing anterior cruciate ligament rupture in a child with open physes. 
Postoperative rehabilitation involved immediate weight bearing, passive motion, and closed chain exercise. Clinical controls had been prepared 30, 60, 90, 150, and 180 days after surgery. Radiological assessments (standard radiograph), Orthopädische Arbeitsgruppe Knie score [27] and KT 1000 [28], have been taken in all patients, 1 year after surgery. At the same time, five of them underwent MRI (Fig. 2).

\section{Results}

ACL lesion was confirmed by arthroscopy in all 10 patients. In the diagnostic part of surgery, one lateral and two medial meniscal tears and one lateral were noticed. Three partial meniscectomies have then been performed.

Physical examination at the follow-up showed that all patients had full range of passive and active motion by comparing the operated knees to controlateral. In addition, Lachman and Pivot shift test was negative in all of them.

During clinical examination, there were no occurrences of growth disturbance, significant $(>1 \mathrm{~cm})$ leg-length inequality, or angular deformity in any of the surgically treated young patients. Furthermore, there were no bony abnormalities in either the patella or the tibial tubercle referable to the harvesting of the bone-patellar tendon bone autograft.

At the 1-year follow-up, radiographies showed that seven of 10 patients have reached skeletal maturity of tibial and femoral physes (Fig. 3). Three patients were nearing skeletal maturity. These three have been checked once more with radiography 2 years after surgery and showed to have reached skeletal maturity. Radiographic examination revealed no evidence of varus or valgus angulation, limblength discrepancy, or early physeal arrest in all patients.

One year after surgery, Orthopädische Arbeitsgruppe Knie score showed excellent results in three patients, good in six, and fair in one patient (medium rate, 87.7). K'T-1000 Arthrometer (Medmetric Corp., San Diego, California, USA) measurements (side-to-side difference) revealed isometric reconstruction in all the knees (Table 1).

Mean follow-up has been 24.9 months (range, 15-44 months). All patients have returned to their preinjury level of daily activity and athletic participation. One patient underwent a second arthroscopy because of a meniscal internal tear, but the ACL was well placed and tensioned (Fig. 4).

One new rupture of the ACL associated with a meniscal medial tear was noticed due to a distorsional trauma in a soccer player. The ACL has been reconstructed again in this patient using allograft, and partial meniscectomy has been performed.

\section{Discussion}

ACL lesion in children and adolescents is a serious injury and its frequency is increasing. The traditional nonoperative treatment of ACL tears in children often leads to unsatisfactory results. Activity modification in the young is often not feasible. The risk of meniscal tears and premature degenerative joint damage in patients with ACL insufficiency are well documented. The literature clearly shows that early, aggressive restoration of knee stability allows the patients to return to their preinjury sport level and decreases the risk of meniscal and cartilage damage [12]. Therefore, ACL reconstruction in skeletally immature patients continues to be a highly controversial topic. The decision to perform a reconstructive ACL procedure in patients with open physes is difficult and has to be well analyzed. As many treatments have been described in the literature, attention must be focused on the choice of the best treatment in relation to the age of the patient $[24,26]$.

A thorough history and a physical examination are crucial in selecting the surgical approach. Assessments of the

Fig. 2
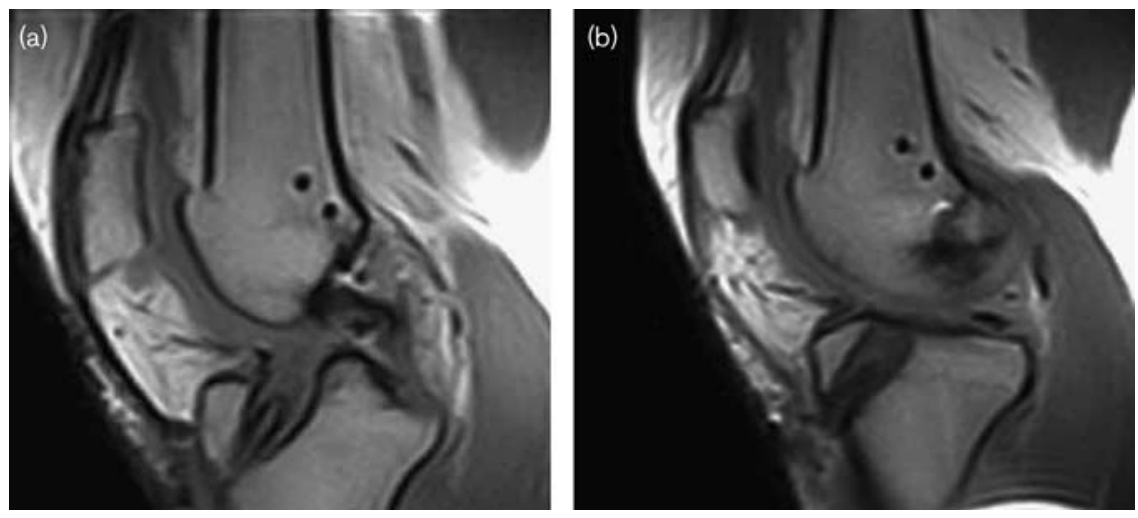

$(a, b)$ Postoperative MRI with anterior cruciate ligament reconstruction tunnel and open physes. 
Fig. 3
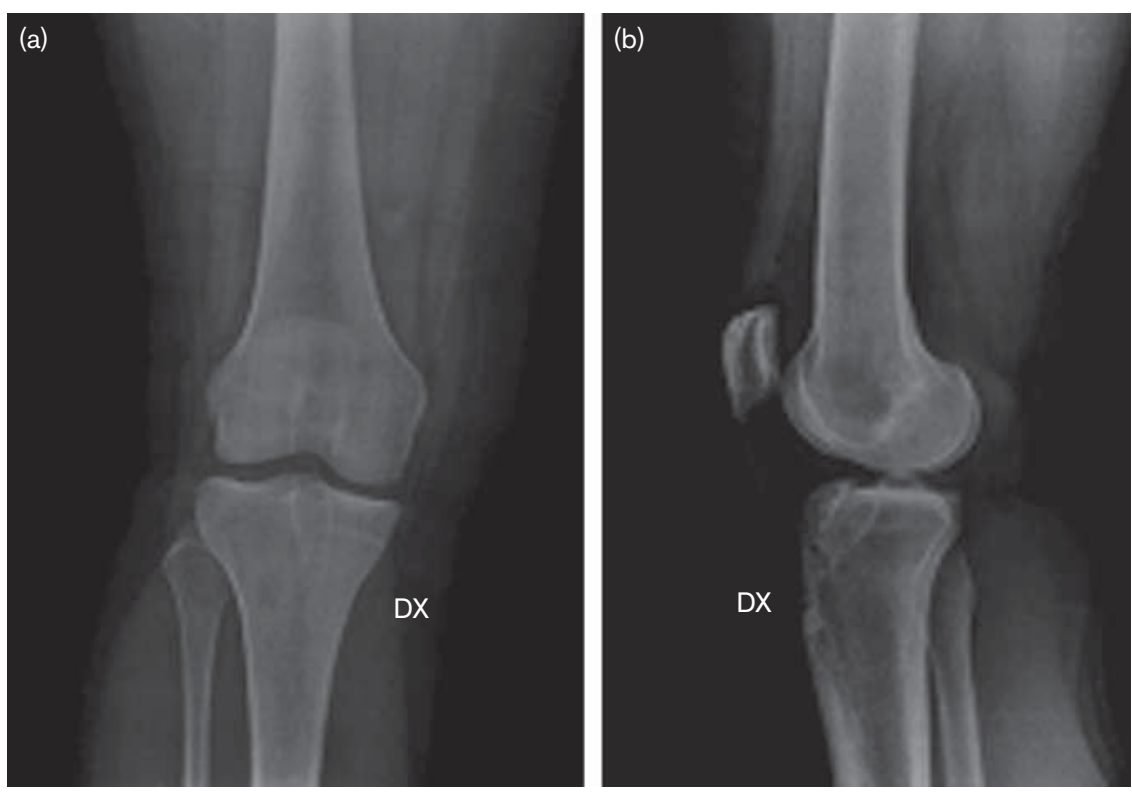

$(a, b)$ Postoperative radiograph of knee joint in two planes showing tunnel and closed physes.

Table 1 Group study

\begin{tabular}{|c|c|c|c|c|c|}
\hline Patient & Age (years) & Procedure & Follow-up (months) & OAK score & $\mathrm{KT}-1000(\mathrm{~mm})^{\mathrm{d}}$ \\
\hline $1^{\mathrm{a}}$ & 13 & $\mathrm{ACL}$ reconstruction + medial meniscal & 44 & 94 (excellent) & 0.7 \\
\hline $2^{b}$ & 14 & $A C L$ reconstruction & 24 & 86 (good) & 3.6 \\
\hline 3 & 14 & $\mathrm{ACL}$ reconstruction & 24 & 85 (good) & 3.3 \\
\hline 4 & 15 & ACL reconstruction & 18 & 86 (good) & 4 \\
\hline 5 & 16 & ACL reconstruction + medial meniscal & 15 & 89 (good) & 3.2 \\
\hline $6^{a}$ & 13 & ACL reconstruction & 42 & 92 (excellent) & 2.1 \\
\hline $7^{\mathrm{a}}$ & 14 & $A C L$ reconstruction & 28 & 83 (good) & 2.8 \\
\hline 8 & 15 & ACL reconstruction & 22 & 88 (good) & 6 \\
\hline $9^{c}$ & 16 & $\mathrm{ACL}$ reconstruction + lateral meniscal & 15 & 78 (fair) & 5.2 \\
\hline 10 & 14 & $\mathrm{ACL}$ reconstruction & 17 & 96 (excellent) & 1.7 \\
\hline
\end{tabular}

$\mathrm{ACL}$, anterior cruciate ligament; OAK, Orthopädische Arbeitsgruppe Knie.

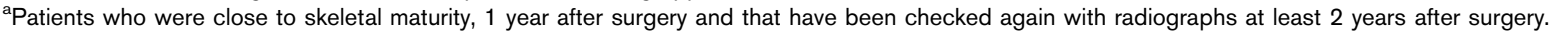

${ }^{b}$ Patient who underwent a second arthroscopy for a new medial meniscal tears.

${ }^{\mathrm{c}}$ Patient who underwent a second arthroscopy for a new medial meniscal tear associated to the rupture of the new ACL.

${ }^{\mathrm{d}}$ Arthrometer testing was performed as manual maximum test involved minimum noninvolved knee.

remaining growth grading in children with the Tanner classification and radiology are important. If we focus our attention on adolescents with growth remaining (boys: 13-16 years; girls: 12-14 years), primary repair and extraarticular procedures are not recommended [10,11]. Intraarticular, however, extraphyseal techniques might be used in the treatment of ACL lesion in children and adolescents with wide-open physes and this seems to be a good option [16,17,19,26,29]. However, some investigators still report that the implant is not isometric [21] and anatomic [22], whereas others demonstrate a low rate of growth damage $[19,20]$. The risk of damaging open physes is connected to the growth age and the choice of the procedure [7-9].
The complete restoration of knee stability can be obtained with an intra-articular complete transphyseal reconstruction of ACL, which is the only real anatomic, isometric procedure [30]. Some investigators have already described the successful transphyseal ACL reconstruction with autogenous hamstrings underlining with minimal risk of growth disturbance and a low revision rate $[23,24]$.

Shelbourne [30] described that the reconstruction with patellar tendon autograft yields good results, but no information is available about the inclination of the tibial tunnel.

The purpose of this study was to report our results after intra-articular transphyseal ACL reconstruction using bone-patellar tendon bone autograft in Tanner 3 patients. 
Fig. 4

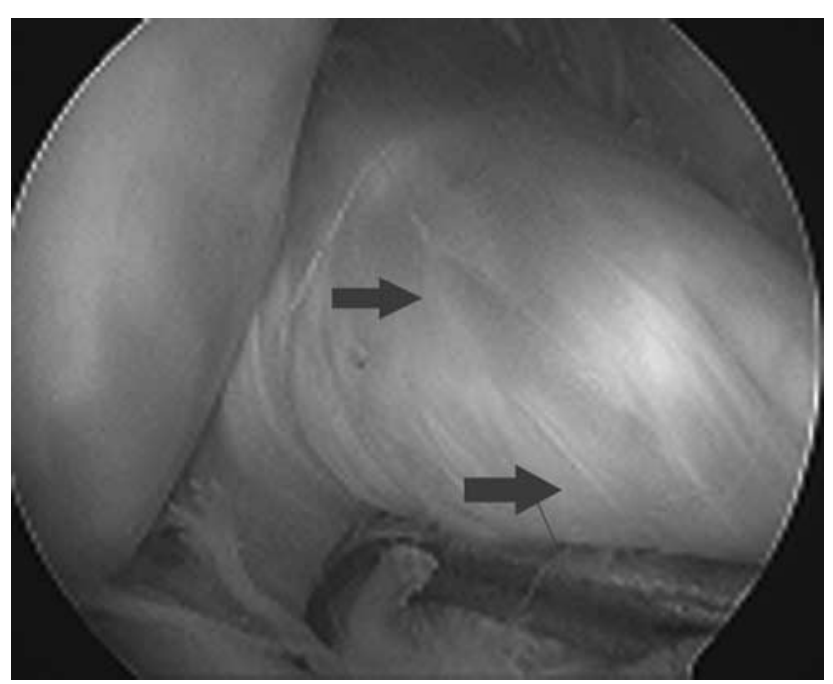

Arthroscopic view of neoligament in a second look operation.

Our study is focused on the importance of the placement of the tibial tunnel. The influence of the diameter of the tunnel on the potential damages of the growth plate is well known $[18,25,26]$. The importance of the angle of the tibial tunnel has been pointed out only by radiological studies, which have underlined that the more the tunnel is drilled vertically, the lower is the risk of potential damages $[25,26]$.

This is the reason why we decided to drill holes with a diameter of less than $9 \mathrm{~mm}$ and with the compass settled between $50^{\circ}$ and $55^{\circ}$. In accordance with the literature, we conclude that to minimize the risk of damaging the open physes, it is necessary to perform central tunnel of less than $10 \mathrm{~mm}$ [18]. We also point out that drilling more vertical tunnels $\left(50-55^{\circ}\right)$ when bone-tendon-bone autograft has been chosen to avoid partial epiphysiodesis, gives good functional and isometric results [25,26]. All our patients returned to their preinjury level of activity and athletic participation. No patient had evidence of early physeal arrest, angulation deformity, or leg-length discrepancy.

These outcomes suggest that skeletally immature athletes with symptomatic ACL tears who do not want to modify their activity level until skeletal maturity may benefit from intra-articular, complete transphyseal ACL reconstruction using bone-patellar tendon bone autograft [31-33].

A different attention should be devoted to very young patients ( $<11$ years), with Tanner stage 1 or 2 and significant skeletal growth prediction. In these cases, we believe activity limitation and rehabilitation are necessary until they reach at least Tanner stage 3 or a skeletal age of 13 years. Attention on bone plug and interference screw placement into metaphyseal bone will help reduce the risk of early physeal arrest [31,32].

\section{Acknowledgements Conflicts of interest}

There are no conflicts of interest.

\section{References}

1 Aichroth PM, Patel DV, Zorrilla P. The natural history and treatment of rupture of the anterior cruciate ligament in children and adolescents: a prospective review. Bone Joint Surg Br 2002; 84:38-41.

2 Guzzanti V. The natural history and treatment of rupture of the anterior cruciate ligament in children and adolescents. J Bone Joint Surg $\mathrm{Br} 2003$; 85:618-619.

3 Dejour H, Neyret P, Eberhard P, Walch G. Bilateral congenital absence of the anterior cruciate ligament and the internal menisci of the knee: a case report. Chir Orthop Reparatrice Appar Mot 1990; 76:329-332.

4 Barrett GR, Tomasin JD. Bilateral congenital absence of the anterior cruciate ligament. Orthopedics 1988; 11:431-434.

5 Paletta GA Jr. Special considerations: anterior cruciate ligament reconstruction in the skeletally immature. Orthop Clin North Am 2003; 34:65-77.

6 Andrish JT. Anterior cruciate ligament injuries in the skeletally immature patient. Am J Orthop 2001; 30:103-110.

7 Lo IK, Bell DM, Fowler PJ. Anterior cruciate ligament injuries in the skeletally immature patient. Instr Course Lect 1998; 47:351-359.

8 Bales CP, Guettler JH, Moorman CT III. Anterior cruciate ligament injuries in children with open physes: evolving strategies of treatment. Am J Sports Med 2004; 32:1978-1985.

9 Schachter AK, Rokito AS. ACL injuries in the skeletally immature patient. Orthopedics 2007; 30:365-370.

10 Mizuta H, Kubota K, Shiraishi M, Otsuka Y, Nagamoto N, Takagi K. The conservative treatment of complete tears of the anterior cruciate ligament in skeletally immature patients. J Bone Joint Surg $\mathrm{Br} 1995$; 77:890-894.

11 Shelbourne KD, Patel DV, McCarroll JR. Management of anterior cruciate ligament injuries in skeletally immature adolescents. Knee Surg Sports Traumatol Arthrosc 1996; 4:68-74.

12 Millett PJ, Willis AA, Warren RF. Associated injuries in pediatric and adolescent anterior cruciate ligament tears: does a delay in treatment increase the risk of meniscal tear? [Review]. Arthroscopy 2002; 18:955-959

13 DeLee JC, Curtis R. Anterior cruciate ligament insufficiency in children. Clin Orthop Relat Res 1983; 172:112-118.

14 McCarroll JR, Rettig AC, Shelbourne KD. Anterior cruciate ligament injuries in the young athlete with open physes. [Review]. Am J Sports Med $1988 ; 16: 44-447$.

15 Lawrence JT, Bowers AL, Belding J, Cody SR, Ganley TJ. All-epiphyseal anterior cruciate ligament reconstruction in skeletally immature patients. Clin Orthop Relat Res 2010; 468:1971-1977.

16 Guzzanti V, Falciglia F, Stanitski CL. Physeal-sparing intraarticular anterior cruciate ligament reconstruction in preadolescents. Am J Sports Med 2003; 31:949-953.

17 Bisson LJ, Wickiewicz T, Levinson M, Warren R. ACL reconstruction in children with open physes. Orthopedics 1998; 21:659-663.

18 Simonian PT, Metcalf MH, Larson RV. Anterior cruciate ligament injuries in the skeletally immature patient. Am J Orthop 1999; 28:624-628.

19 Kocher MS, Garg S, Micheli LJ. Physeal sparing reconstruction of the anterior cruciate ligament in skeletally immature prepubescent children and adolescents: surgical technique. J Bone Joint Surg Am 2006; 88 (Suppl 1 Pt 2):283-293.

20 Finlayson CJ, Nasreddine A, Kocher MS. Current concepts of diagnosis and management of ACL injuries in skeletally immature athletes. Phys Sportsmed 2010; 38:90-101.

21 Nakhostine M, Bollen SR, Cross MJ. Reconstruction of mid-substance anterior cruciate rupture in adolescents with open physes. $J$ Pediatr Orthop 1995; 15:286-287.

22 Parker AW, Drez D Jr, Cooper JL. Anterior cruciate ligament injuries in patients with open physes. Am J Sports Med 1994; 22:44-47.

23 Cohen M, Ferretti M, Quarteiro M, Marcondes FB, de Hollanda JP, Amaro JT, Abdalla RJ. Transphyseal anterior cruciate ligament reconstruction in patients with open physes. Arthroscopy 2009; 25:831-838.

24 Kocher MS, Smith JT, Zoric BJ, Lee B, Micheli LJ. Transphyseal anterior cruciate ligament reconstruction in skeletally immature pubescent adolescents. J Bone Joint Surg Am 2007; 89:2632-2639.

25 Shea KG, Apel PJ, Pfeiffer RP, Traughber PD. The anatomy of the proximal tibia in pediatric and adolescent patients: implications for $\mathrm{ACL}$ 
reconstruction and prevention of physeal arrest. Knee Surg Sports Traumatol Arthrosc 2007; 15:320-327.

26 Kercher J, Xerogeanes J, Tannenbaum A, Al-Hakim R, Black JC, Zhao J. Anterior cruciate ligament reconstruction in the skeletally immature: an anatomical study utilizing 3-dimensional magnetic resonance imaging reconstructions. J Pediatr Orthop 2009; 29:124-129.

27 Müller W, Biedert R, Hefti F, Jakob RP, Munzinger U, Stäubli HU. OAK knee evaluation: a new way to assess knee ligament injuries. Clin Orthop Relat Res 1988; 232:37-50.

28 Rangger C, Daniel DM, Stone ML, Kaufman K. Diagnosis of an ACL disruption with KT-1000 arthrometer measurements. Knee Surg Sports Traumatol Arthrosc 1993; 1:60-66.

29 Andrews M, Noyes FR, Barber-Westin SD. Anterior cruciate ligament allograft reconstruction in the skeletally immature athlete. Am J Sports Med 1994; 22:48-54.
30 Shelbourne KD, Gray T, Wiley BV. Results of transphyseal anterior cruciate ligament reconstruction using patellar tendon autograft in Tanner stage 3 or 4 adolescents with clearly open growth plates. Am J Sports Med 2004; 32:1218-1222.

31 Fuchs R, Wheatley W, Uribe JW, Hechtman KS, Zvijac JE, Schurhoff MR. Intra-articular anterior cruciate ligament reconstruction using patellar tendon allograft in the skeletally immature patient. Arthroscopy 2002; 18:824-828.

32 Lo IK, Bell DM, Fowler P. Anterior cruciate ligament injuries in the skeletally immature patient [Review]. Instr Course Lect 1998; 47: 351-359.

33 Lo IK, Kirkley A, Fowler PJ, Miniaci A. The outcome of operatively treated anterior cruciate ligament disruptions in the skeletally immature child. Arthroscopy 1997; 13:627-634. 